\title{
ON TOPOLOGICALLY INDUCED GENERALIZED PROXIMITY RELATIONS
}

\author{
MICHAEL W. LODATO
}

1. Introduction. In this paper we examine one of the results in the theory of the proximity spaces of Efremovic [1]:

$A$ set $X$ with a binary relation " $A$ close to $B$ " (written $A \delta B$ ) is a proximity space if and only if there exists a compact Hausdorff space $Y$ in which $X$ can be topologically imbedded so that

$$
A \delta B \text { in } X \text { if and only if } \bar{A} \text { meets } \bar{B} \text { in } Y
$$

( $\bar{A}$ denotes the closure of the set $A$ ) [2].

This proposition raises the question: Can we characterize the relations $\delta$ for which this result holds under weaker conditions on $Y$ ? In $\$ 4$ we give an affirmative answer (Theorem 5.3) using rather mild restrictions on $Y$ and on the imbedding of $X$ in $Y$. This result is essentially a corollary to a fundamental theorem (Theorem 4.2).

2. Symmetric generalized proximity spaces. As in [3] we define a symmetric generalized proximity space (or $P_{\mathrm{s}}$-space) to be an abstract set $X$ with a binary operation " $A \delta B$ " (a $P_{s}$-relation) on its power set satisfying the following axioms:

(P.1) $A \delta(B \cup C)$ implies that either $A \delta B$ or $A \delta C$.

(P.2) $A \delta B$ implies that $A$ and $B$ are nonvoid.

(P.3) If $A$ meets $B$ then $A \delta B$.

(P.4) $A \delta B$ and $b \delta C$ for all $b$ in $B$ implies that $A \delta C$.

(P.5) $A \delta B$ implies $B \delta A$.

We read the symbols " $A \delta B$ " as " $A$ is close to $B$ "; and we say that " $A$ is remote from $B$ " (in symbols, " $A$ not $\delta B$ ") if $A$ is not close to $B$.

(2.1) The following facts are evident: (1) If $A \delta B, A \subset C$, and $B \subset D$, then $C \delta D$. (2) Define

$$
A^{\delta}=\{x \in X: x \delta A\}
$$

then in a $P_{s}$-space $\left(A^{\delta}\right) \delta\left(B^{\delta}\right)$ if and only if $A \delta B$.

(2.2) In [3] it is shown that there is a topology induced on every $P_{\text {s-space }}(X, \delta)$ by the closure operation $A \rightarrow A^{\delta}$. Moreover, this topology is symmetric: $x$ in $\bar{y}$ implies $y$ in $\bar{x}$ for all points $x, y \in X$. Clearly, every $T_{1}$ topological space is symmetric.

(2.3) Theorem. Given any symmetric topological space $X$ define $\delta_{0}$ by:

Received by the editors October 13, 1962 and, in revised form, February 9, 1963. 

$A \delta_{0} B$ if and only if $\bar{A}$ meets $\bar{B}$.

Then $\delta_{0}$ is a $P_{\mathrm{s}}$-relation and is compatible with the given topology: $x \delta_{0} B$ if and only if $x \in \bar{B}$.

Proof. We derive axioms (P.1) through (P.5) by use of the Kuratowski closure axioms [4]. Axioms (P.1), (P.2), (P.3) and (P.5) are trivial results of the closure axioms and (2.4). For (P.4), note that if for a point $b$ and a set $C$ we have $\bar{b} \cap \bar{C} \neq \varnothing$, then there exists a point $c$ in $C$ such that $c \in \bar{b}$. By symmetry then $b \in \bar{c} \subset \bar{C}$. Thus, if $\bar{A} \cap \bar{B} \neq \varnothing$ and $\bar{b} \cap \bar{C} \neq \varnothing$ for every $b$ in $B$ then $\bar{B} \subset \bar{C}$ and so $\bar{A} \cap \bar{C} \neq \varnothing$. It is now clear, from the above argument, that $\delta_{0}$ is compatible with the given topology.

(2.5) Theorem. Given a $P_{s}$-space $(X, \delta)$ and $\delta_{0}$ defined by $(2.4)$ in terms of the topology induced by $\delta$ we have that $A \delta_{0} B$ implies that $A \delta B$ for all subsets $A$ and $B$ of $X$. Thus $\delta_{0}$ is the smallest P-relation compatible with the topology in a symmetric topological space.

Proof. The demonstration follows directly from (2.3), (2.1) and (P.3).

3. Clusters. A cluster $\pi$ from a $P_{8}$-space $(X, \delta)$ is a class of subsets of $X$ satisfying:

(C.1) $A \delta B$ for all $A, B \in \pi$.

(C.2) $A \cup B \in \pi$ implies that either $A \in \pi$ or $B \in \pi$.

(C.3) If $B \delta A$ for every $A$ in $\pi$, then $B \in \pi$.

Note that this is the same definition used by Leader [5] in introducing clusters for Efremovic proximity spaces.

(3.1) Theorem. For $x$, a point in a $P_{s}$-space $(X, \delta)$, the class $\pi_{x}$ of all subsets of $X$ which are close to $x$ is a cluster from $X$.

Proof. We must show that $\pi_{x}$ satisfies (C.1), (C.2) and (C.3). For (C.1) suppose $A, B \in \pi_{x}$. Then $x \delta A$ and $x \delta B$ so that, by (2.5), $A \delta B$. For (C.2) suppose $A \cup B \in \pi_{x}$. Then $x \delta(A \cup B)$ and, by (P.1), this means that either $x \delta A$ or $x \delta B$, that is, either $A \in \pi_{x}$ or $B \in \pi_{x}$. For (C.3) suppose that $A \delta C$ for every $C$ in $\pi_{x}$. Since, by (P.3), $\{x\} \in \pi_{x}$, we have in particular, that $A \delta x$ or, $A \in \pi_{x}$.

(3.2) The following facts are easily established. (1) Any cluster $\pi$ from a $P_{\mathrm{z}}$-space $(X, \delta)$ is closed under the operation of supersets: if $\pi$ is a cluster from $X, A \in \pi$, and $A \subset B$, then $B \in \pi$. (2) If $A \in \pi$, a cluster from $X$, and $a \delta B$ for every $a$ in $A$, then $B \in \pi$. (3) If $\pi$ and $\pi^{\prime}$ are clusters from $X$ and $\pi$ is a subclass of $\pi^{\prime}$, then $\pi=\pi^{\prime}$. (4) If a point $x$ belongs to a cluster $\pi$, then $\pi$ is just the class $\pi_{x}$ of all subsets $A$ of $X$ such that $A \delta x$. (5) Given a cluster $\pi$ from a nonvoid $P_{s}$-space 
$(X, \delta)$ and any subset $A$ of $X$, then either $A \in \pi$ or $X-A \in \pi$. (6) Let $\pi$ be a cluster from $(X, \delta)$. If $A$ is a subset of $X$ which meets every member of $\pi$, then $A \in \pi$.

4. Extensions characterized by clusters. We say that a subset $X$ of a topological space $Y$ is regularly dense in $Y$ if and only if given $U$ open in $Y$ and $p$ a point in $U$ there exists a subset $E$ of $X$ with $p \in \bar{E} \subset U$, the closure being taken in $Y$.

(4.1) Theorem. If $X$ is regularly dense in $Y$, then $X$ is dense in $Y$. If $Y$ is regular and $X$ is dense in $Y$ then $X$ is regularly dense in $Y$.

Proof. $Y$ is open in $Y$, hence for any point $p$ in $Y$ there exists a subset $E$ of $X$ such that $p \in \bar{E} \subset \bar{X} \subset Y$. Since this is true for any $p$ in $Y$, we have $Y \subset \bar{X} \subset Y$.

For $Y$ regular, $y \in Y$ and $U$ an open set of $Y$ containing $y$ we have the existence of an open set $V$ of $Y$ containing $y$ such that $\bar{V} \subset U$. Now $E=V \cap X$ is a subset of $X$ and $\bar{E}=\mathrm{Cl}(V \cap X)=\bar{V} \subset U,{ }^{1}$ with the second equality following from the density of $X$ in $Y$. Thus, $y \in \bar{E} \subset U$.

(4.2) TheOREM. Given $a$ set $X$ and some binary relation $\delta$ on the power set of $X$, the following are equivalent:

(I) There exists a $T_{1}$ topological space $Y$ and a mapping $f$ of $X$ into $Y$ such that $f X$ is regularly dense in $Y$ and

$A \delta B$ in $X$ if and only if $\mathrm{Cl}(f A)$ meets $\mathrm{Cl}(f B)$ in $Y$.

(II) $\delta$ is a $P_{\mathrm{s}}$-relation satisfying the additional axiom,

(P.6) Given $A \delta B$ in $X$ there exists a cluster $\pi$ to which both $A$ and $B$ belong.

Proof. Suppose that (I) holds and define $\delta$ by (4.3). (P.1), (P.2), (P.3) and (P.5) are trivial consequences of the properties of closure. For (P.4) suppose that $A \delta B$ and $b \delta C$ for all $b$ in $B$. Then $\mathrm{Cl}(f A)$ $\cap \mathrm{Cl}(f B) \neq \varnothing$ and $\mathrm{Cl}(f b) \cap \mathrm{Cl}(f C) \neq \varnothing$ for all $b$ in $B$, which since $Y$ is $T_{1}$, implies that $f b \in \mathrm{Cl}(f C)$ for all $b$ in $B$. Thus $f B C \mathrm{Cl}(f C)$ or $\mathrm{Cl}(f B) \subset \mathrm{Cl}(f C)$ so that $\mathrm{Cl}(f A) \cap \mathrm{Cl}(f C) \neq \varnothing$ showing that $A \delta C$. For (P.6), since $\mathrm{Cl}(f A) \cap \mathrm{Cl}(f B) \neq \varnothing$, let $c \in \mathrm{Cl}(f A) \cap \mathrm{Cl}(f B)$ and define $\pi$ to be the class of all subsets $S$ of $X$ such that $c \in \mathrm{Cl}(f S)$. Clearly $A$ and $B$ are in $\pi$ and in showing that $\pi$ is a cluster the demonstrations of (C.1) and (C.2) are trivial. For (C.3) suppose that $\mathrm{Cl}(f D) \cap \mathrm{Cl}(f C) \neq \varnothing$ for every $C$ in $\pi$ but that $D \notin \pi$, i.e., $c \notin \mathrm{Cl}(f D)$. Thus, $c \in Y-\mathrm{Cl}(f D)$ and since $F X$ is regularly dense in $Y$ there exists a subset $E$ of $X$ such that $c \in \mathrm{Cl}(f E) \subset Y-\mathrm{Cl}(f D)$. That is, there

${ }^{1}$ Where $\mathrm{Cl}$ stands for closure. 
exists an $E$ in $\pi$ such that $\mathrm{Cl}(f D) \cap \mathrm{Cl}(f E)=\varnothing$. This contradicts the hypothesis of (C.3). Thus (II) is satisfied.

For the converse suppose that (II) holds. Given $x$ in $X$ the class $\pi_{x}$ of all subsets $A$ of $X$ such that $x \delta A$ is a cluster from $X$, by (3.1). Thus for any subset $A$ of $X$, let $a$ be the set of all clusters $\pi_{a}$ determined by the points $a$ in $A$. Let $\bar{a}$ be the set of all clusters to which $A$ belongs. By (P.3), $A \in \pi_{a}$ for each $a$ in $A$ and so $a \subset \bar{a}$. We will denote $\bar{X}$, the set of all clusters from $X$, by $Y$.

A subset $A$ of $X$ absorbs a subset $\beta$ of $Y$ if and only if $A$ belongs to every cluster in $\beta$, that is, if and only if $\bar{a}$ contains $\beta$. For any subset $\beta$ of $Y$ we define the closure, $\operatorname{cl}(\beta)$, of $\beta$ by

(4.4) $\pi \in \operatorname{cl}(\beta)$ if and only if every subset $E$ of $X$ which absorbs $\beta$ is in $\pi$.

We next show that

(4.5) $\operatorname{cl}(a)=\bar{a}$.

For if $\pi \in \operatorname{cl}(a)$ then since $A$ absorbs $a, A \in \pi$ so that $\pi \in \bar{a}$. On the other hand, if $\pi \in \bar{a}$ then $A \in \pi$. Now let $P$ be in every $\pi_{a}$ in $Q$, i.e., $P \delta a$ for every $a$ in $A$ and hence $A \subset P^{\delta}$. Thus, by (3.2), (2), $P \in \pi$ so that $\pi \in \operatorname{cl}(a)$.

We now show that the Kuratowski closure axioms are satisfied by the closure defined by (4.4).

(K.1) $\beta \subset \operatorname{cl}(\beta)$ : This is trivial since if $E$ absorbs $\beta$ then $E \in \pi$ for every $\pi \in \beta$.

(K.2) $\operatorname{cl}(\varnothing)=\varnothing$ : Suppose $\pi \in \operatorname{cl}(\varnothing)$. Since it is vacuously true that every subset of $X$ absorbs $\varnothing$, we then have that every subset of $X$ is in $\pi$. In particular, $\varnothing$ and $X$ are in $\pi$. Thus, $\varnothing \delta X$, by (C:1), contradicting (P.2).

(K.3) $\operatorname{cl}(\operatorname{cl}(\beta)) \subset \operatorname{cl}(\beta)$ : Suppose $\pi \in \operatorname{cl}(\operatorname{cl}(\beta))$ and that $E$ absorbs $\beta$. By (4.4), $E$ absorbing $\beta$ implies that $E$ absorbs $\operatorname{cl}(\beta)$. Hence $E \in \pi$ showing that $\pi \in \operatorname{cl}(\beta)$.

(K.4) $\operatorname{cl}\left(\beta \cup \beta^{\prime}\right)=\operatorname{cl}(\beta) \cup \operatorname{cl}\left(\beta^{\prime}\right)$ : Suppose that $\pi \in \operatorname{cl}\left(\beta \cup \beta^{\prime}\right)$ and that $A$ absorbs $\beta$ and $A^{\prime}$ absorbs $\beta^{\prime}$. Then, by (3.2), (1), $A \cup A^{\prime}$ absorbs $\beta \cup \beta^{\prime}$ so that $A \cup A^{\prime} \in \pi$. But, by (C.2), this means that either $A \in \pi$ or $A^{\prime} \in \pi$, that is $\pi \in \operatorname{cl}(\beta)$ or $\pi \in \operatorname{cl}\left(\beta^{\prime}\right)$. Thus $\pi \in \operatorname{cl}(\beta) \cup \operatorname{cl}\left(\beta^{\prime}\right)$ and we have $\operatorname{cl}\left(\beta \cup \beta^{\prime}\right) \subset \operatorname{cl}(\beta) \cup \operatorname{cl}\left(\beta^{\prime}\right)$. On the other hand, $\pi \in \operatorname{cl}(\beta) \cup \operatorname{cl}\left(\beta^{\prime}\right)$ implies that either $\pi \in \operatorname{cl}(\beta)$ or $\pi \in \operatorname{cl}\left(\beta^{\prime}\right)$. Now if $E$ absorbs $\beta \cup \beta^{\prime}$, then $E$ absorbs $\beta$ and also absorbs $\beta^{\prime}$. Hence, $E \in \pi$ showing that $\pi \in \operatorname{cl}\left(\beta \cup \beta^{\prime}\right)$ and (K.4) holds.

To show that the topology is $T_{1}$, suppose $\pi^{\prime} \in \operatorname{cl}(\pi)$, where $\pi$ and $\pi^{\prime}$ are clusters from $X$. This means that every set in $\pi$ is also in $\pi^{\prime}$. Thus, $\pi \subset \pi^{\prime}$ and by (3.2), (3), $\pi=\pi^{\prime}$. Hence, $\operatorname{cl}(\pi)=\pi$ for every point $\pi$ in the space $Y$. 
Now the correspondence which assigns to each point $x$ in $X$ the cluster $\pi_{x}$ determined by it is a well-defined transformation mapping $X$ into $Y$ which we will denote by $f$. Note that $f A=Q$ for every subset $A$ of $X$, so in order to show that (4.3) holds we must show that, using $(4.5)$,

$$
A \delta B \text { in } X \text { if and only if } \bar{Q} \text { meets } \bar{B} \text { in } Y \text {. }
$$

So if $A \delta B$ there exists, by (P.6), a cluster $\pi$ to which both $A$ and $B$ belong. Thus, by definition of $\bar{a}$, we have $\pi \in \bar{a} \cap \bar{B}$. On the other hand, if $\pi \in \bar{Q} \cap \bar{B}$ then $A$ and $B$ are in $\pi$ so that, by (C.1), $A \delta B$.

To show that $f X=x$ is regularly dense in $Y$ suppose that $\alpha$ is an open subset of $Y$ and that $\pi \in \alpha$. We thus have $\pi \in Y-\alpha=\operatorname{cl}(Y-\alpha)$. This means, by (4.4), that there exists some subset $E$ of $X$ such that $E$ is in every cluster of $Y-\alpha$ but that $E$ is not in $\pi$. Hence, by (C.3), there is a $C$ in $\pi$ such that $E$ not $\delta C$.

Since $\overline{\mathrm{C}}$ is the set of all clusters to which $C$ belongs we have $\pi \in \overline{\mathrm{e}}$. And since $E$ belongs to every cluster in $Y-\alpha$ and $E$ not $\delta C$, then $C$ cannot belong to any cluster in $Y-\alpha$, by (C.1). Hence $\overline{\mathfrak{e}}$ is contained in $\alpha$ and we have shown that $X$ is regularly dense in $Y$.

The proof is now complete.

5. Symmetric $P_{1}$-spaces. A $P_{s}$-space $(X, \delta)$ in which $\delta$ satisfies the additional axiom

$$
x \delta y \text { implies } x=y \text { for all points } x, y \in X
$$

is called a symmetric $P_{1-s p a c e}$ (see [3]). The following theorem follows directly from (C.1) and (5.1).

(5.2) Theorem. Every cluster $\pi$ from a symmetric $P_{1}$-space $(X, \delta)$ possesses at most one point.

(5.3) Theorem. Given a set $X$ and some binary relation $\delta$ on the power set of $X$, the following are equivalent:

$\left(\mathrm{I}^{\prime}\right)$ There exists a $T_{1}$ topological space $Y$ in which $X$ can be topologically imbedded as a regularly dense subset so that (1.1) holds.

$\left(\mathrm{II}^{\prime}\right) \delta$ is a symmetric $P_{1}$-relation satisfying (P.6).

Proof. The demonstration is similar to that of Theorem (4.2). To see that (5.1) holds, note that $\bar{x} \cap \bar{y} \neq \varnothing$ implies that $x \cap y \neq \varnothing$, or $x=y$.

To show that our imbedding is topological we note first that, because of (5.2) the correspondence between $X$ and $x$ induced by the identification of $x$ with the cluster $\pi_{x}$ determined by it is one-to-one. To see that the mapping is bicontinuous we must show that if $A$ is a 
subset of $X, x \in A^{\delta}$ if and only if $\pi_{x} \in \mathrm{kl}(Q)$, where $\mathrm{kl}(Q)$ is the closure of $a$ in $X$ relative to the space $Y$.

So suppose $x \in A^{\delta}$ and that $P$ absorbs $a$. Then $P$ is a member of every $\pi_{a}$ in $a$ and it follows that $a \delta P$ for every $a$ in $A$. Thus, $A \subset P^{\delta}$ and since $A \in \pi_{x}$ we have, from (3.2), (2), that $P \in \pi_{x}$. Thus, $\pi_{x} \in \mathrm{kl}(Q)$.

On the other hand, suppose $\pi_{x} \in \mathrm{kl}(Q)$. Then since $A$ absorbs $Q$ we have $A \in \pi_{x}$, i.e., $A \delta x$ and hence $x \in A^{\delta}$. This completes the proof.

\section{REFERENCES}

1. V. A. Efremovic, The geometry of proximity, Mat. Sb. (N.S.) 31 (73) (1952), 189-200.

2. Yu. M. Smirnov, On proximity spaces, Mat. Sb. (N.S.) 31 (73) (1952), 543-574.

3. M. W. Lodato, Generalized proximity spaces: A generalization of topology, (to appear).

4. K. Kuratowski, Sur l'operation A de l'Analysis Situs, Fund. Math. 3 (1922), 182-199.

5. S. Leader, On clusters in proximity spaces, Fund. Math. 47 (1959), 205-213.

Laboratory for Electronics, Inc., Monterey, California 